OPEN ACCESS

Edited by:

Sophie Hillion,

INSERM U1227 Lymphocytes B et Autoimmunite (INSERM), France

Reviewed by:

Terry Francis Davies,

Icahn School of Medicine at Mount

Sinai, United States

Weiping Teng,

First Affiliated Hospital of Anhui

Medical University, China

*Correspondence: Huiyong Peng

penghuiyong33815@163.com Yingzhao Liu

zjliuyingzhao@ujs.edu.cn

Specialty section: This article was submitted to Autoimmune and

Autoinflammatory Disorders,

a section of the journal

Frontiers in Immunology

Received: 12 August 2021

Accepted: 29 October 2021

Published: 15 November 2021

Citation:

Zou J, Peng H and Liu Y (2021) The

Roles of Exosomes in

Immunoregulation and Autoimmune

Thyroid Diseases.

Front. Immunol. 12:757674.

doi: 10.3389/fimmu.2021.757674

\section{The Roles of Exosomes in Immunoregulation and Autoimmune Thyroid Diseases}

\author{
Junli Zou ${ }^{1}$, Huiyong Peng ${ }^{2,3^{*}}$ and Yingzhao Liu ${ }^{1 *}$ \\ ${ }_{1}^{1}$ Department of Endocrinology, The Affiliated People's Hospital of Jiangsu University, Zhenjiang Medical School of Nanjing \\ Medical University, Zhenjiang, China, ${ }^{2}$ Department of Laboratory Medicine, The Affiliated People's Hospital of Jiangsu \\ University, Zhenjiang Medical School of Nanjing Medical University, Zhenjiang, China, ${ }^{3}$ Department of Genetic Toxicology, \\ The Key Laboratory of Modern Toxicology of Ministry of Education, Center for Global Health, School of Public Health, \\ Nanjing Medical University, Nanjing, China
}

Exosomes are extracellular microvesicles $(30-150 \mathrm{~nm})$ released from cells that contain proteins, lipids, RNA and DNA. They can deliver bioactive molecules and serve as carriers facilitating cell-cell communication, such as antigen presentation, inflammatory activation, autoimmune diseases (AIDs) and tumor metastasis. Recently, much attention has been attracted to the biology and functions of exosomes in immune regulation and AIDs, including autoimmune thyroid diseases (AITDs). Some studies have shown that exosomes are involved in the occurrence and development of AITDs, but they are still in the preliminary stage of exploration. This review mainly introduces the association of exosomes with immune regulation and emphasizes the potential role of exosomes in AITDs, aiming to provide new research strategies and directions for the pathogenesis and early diagnosis of AITDs.

Keywords: exosomes, immune regulation, Hashimoto's thyroiditis, Graves' disease, autoimmune thyroid diseases (AITD)

\section{INTRODUCTION}

Autoimmune thyroid diseases(AITDs) are thyroid diseases caused by autoimmune disorders, mainly including Hashimoto's thyroiditis (HT) and Graves' disease (GD) (1). This disease is mainly manifested by infiltration of lymphocytes and the production of autoantibodies, among which autoantibodies interfere with thyroid function, leading to hypothyroidism or hyperthyroidism (2). AITDs are usually accompanied by the production of thyroid peroxidase antibodies (TPOAb), thyroglobulin antibodies (TGAb), thyrotropin receptor antibodies (TRAb) and other autoantibodies (3). The pathogenesis of this disease is very complicated and is mainly classified into genetic predisposition, environmental implications, and immunological factors which are particularly important (4). T lymphocytes and their secreted cytokines play an indispensable role in the regulation of immune response (5). Dysfunction of these $\mathrm{T}$ cells or abnormal expression of these cytokines can lead to the destruction of immune tolerance and abnormal immune responses during the development of AITDs. In recent years, the incidence of AITDs has been on the rise, causing adverse effects on people's quality of life, and increasing the risk of some non-thyroid diseases, such as other AIDs (type 1 diabetes, systemic lupus erythematosus, etc.), cardiovascular 
diseases, thyroid cancer, etc. (6). Therefore, it is of great importance to search for early diagnostic markers and effective therapeutic targets of ATIDs.

Recently, the finding that exosomes are involved in AIDs has been a major breakthrough in the field, unveiling their capacity to modulate immune responses. Exosomes are nanoscale extracellular vesicles secreted by various cells that carry specific substances such as proteins, lipids and nucleic acids to conduct cell-to-cell signal transduction, participate in the occurrence of a variety of diseases and may become new biomarkers (7). In AIDs, antigen-presenting cells (APCs) presenting autoantigens can spread MHC (major histocompatibility complex)/polypeptide complexes to secondary lymph nodes by releasing exosomes, indirectly activating more antigen-specific $\mathrm{T}$ cells and aggravating the occurrence and development of AIDs (8). As one of the most prevalent autoimmune diseases, AITDs are caused by an abnormal autoantigen clearance system and an imbalance in immunoregulation and inflammatory mechanism (9). Autoimmune intolerance is one of the leading theories proposed for thyrocyte destruction in AITDs via the imbalance of regulatory $\mathrm{T}$ cells (Tregs) and $\mathrm{T}$ helper 17 (Th17) cells in adaptive immunity (10). Exosomes derived under pathological conditions can influence Treg and Th17 cell balance in the disease microenvironment, which may contribute to the disruption of autoimmune tolerance in AITDs. In this regard, it has been reported that (11) plasma microvesicles can regulate the differentiation of Tregs and Thl7 cells in AITDs, which is related to their cargos such as miRNAs. Therefore, exosomes and their cargos might play a role in immunoregulation and AITD pathogenesis. In this review, we will highlight the current understanding of exosomes in immunoregulation and AITDs and discuss how exosomes may contribute to AITD pathogenesis.

\section{THE BIOGENESIS AND FUNCTIONS OF EXOSOMES}

Exosomes are extracellular vesicles synthesized and secreted by eukaryotic cells and were originally discovered in the supernatant of sheep red blood cells (12). Under a transmission electron microscope, exosomes are 30-150 nanometers in diameter (13), surrounded by bilayer phospholipid molecules (14), in the shape of plates or cups (15), and usually exist in the sucrose density layer of $1.13-1.19 \mathrm{~g} / \mathrm{ml}$ (16). The biogenesis of exosomes starts within the endosomal system and occurs via intracellular cytoplasmic transport pathways involved in multivesicular cell fusion (17). Mathivanan et al. (18) reported the mechanism of exosome formation whereby exosomes originate from early endosomes. First, after endocytosing exogenous substances, cells combine with cytoplasmic proteins, nucleic acids and lipids to form early endosomes, which further mature into multivesicular bodies (MVBs) by a series of intracellular interactions. This is followed by fusion with the cell membrane and the release of intraluminal vesicles (ILVs) out of cells as exosomes $(19,20)$. Exosomes contain multifarious cargos such as proteins, lipids, DNA, messenger RNA (mRNA), and noncoding RNA and other biomolecules (21).
Although exosomes may have similar surface proteins, such as membrane transport proteins, integrins, heat shock proteins (HSP60, HSP70, HSP90) and transmembrane four-protein superfamily (CD9, CD63, CD81, CD82), there is currently no widely accepted specific marker to distinguish exosomes from different cell populations (22). Due to the lipid bilayer structure, exosomes can stably exist in a variety of biological liquids without being degraded and can transfer biologically active substances into recipient cells to mediate the information exchange between cells, playing a key role in multiple diseases $(23,24)$.

Most cell types can actively secrete exosomes (25), such as tumor cells, endothelial cells, immune cells, stem cells, and nerve cells. Moreover, exosomes exist in different body fluids (26), including blood, cerebrospinal fluid, urine, lymph, ascites, bile, saliva, tears, amniotic fluid and breast milk. Exosomes have a wide range of sources and various functions, which are closely related to the cellular origin of exosomes. To date, overwhelming evidence indicates that exosomes from different cells have different biological characteristics and will evoke totally different responses in recipient cells under the influence of their contents, tissue microenvironment, receptor cells and other factors. For example, tumor cell-derived exosomes can participate in tumor metastasis, promote or inhibit tumor progression, and promote angiogenesis (27). Exosomes derived from bone cells have the functions of bone remodeling, bone metabolism, osteoblast and osteoclast differentiation (28). Exosomes derived from mesenchymal stem cells (MSCs) are able to facilitate immune regulation, angiogenesis promotion, information transmission, tissue repair and cell proliferation (29). Exosomes, serving as circulating biomarkers, play pivotal roles in intercellular communication under physiological and pathological conditions, such as immune signal transduction, inflammation, angiogenesis, and tissue repair (30-32). Due to their inherent properties, such as stability, biocompatibility, and invisibility, exosomes have also emerged as promising therapeutic delivery tools. Several studies have investigated the therapeutic potential of exosomes in immunoregulation, revealing that exosomes containing anti-inflammatory molecules can be used as immunomodulators for the treatment of inflammation, hypersensitivity and autoimmune diseases (33). Hence, we believe there is tremendous future potential for the use of exosomes in therapeutic and diagnostic area of diseases and many other applications.

\section{THE ROLES OF EXOSOMES IN THE IMMUNE SYSTEM}

Exosomes are rich in many bioactive molecules (chemokines, inflammatory factors, signal transduction factors, HSPs, cell specific antigens, various RNAs, etc.) or they carry proteins with special functions (adhesion molecules, costimulatory molecules, ligands, receptors, etc.) on their surface. Notably, exosomes contain specific noncoding RNAs, such as microRNAs (miRNAs) and circular RNAs (circRNAs) that can be functionally transferred to target cells, consequently leading to 
immune activation and suppression (34). Similarly, Okoye et al. (35) found that Treg-derived exosomes can transport miRNAs (such as Let-7d) to Th1 cells, leading to immunosuppression and the prevention of systemic disease. Exosomes modulate the immune response mainly through two mechanisms: exosomes directly act on target cells to activate downstream signals and exosomes regulate immunoreactions through exosomal miRNA mediation (36). The former act in three main ways: the direct action of surface signaling molecules, intracellular regulation of signaling molecules during membrane fusion, and extracellular release of bioactive components (37). Current studies have shown that exosomes secreted from tumor cells, stem cells and some exogenously stimulated immune cells can stimulate immune cells and regulate the function of the immune system (Table 1).

At the same time, different immune cells also coordinate the immune process through exosomes (52). Exosomes from various immune cells transfer important information between immune cells and immune cells, as well as between immune cells and target proteins, and regulate innate and adaptive immunity by participating in antigen presentation or transporting substances such as killer proteins and inflammatory factors (53). Cells involved in innate immunity, such as macrophages, DCs, NK cells, and granulocytes, can recognize antigens through a class of pattern recognition receptors (PRRs), thus generating immune responses. Exosomes can affect the polarization of macrophages (54), the regulation and expression of DCs (55), and the killing effect of NK cells $(56,57)$. Adaptive immunity refers to the immune process in which $\mathrm{T}$ and $\mathrm{B}$ cells are activated, proliferate and differentiate into effector cells after receiving antigen stimulation, resulting in a series of biological effects. In adaptive immune responses, exosomes can disseminate antigens or MHC peptide complexes to increase the number of dendritic cells presenting them, or to directly interact with memory T cells (58).

The immunoregulatory effects of exosomes mainly include affecting antigen presentation, $\mathrm{T}$ cell activation, immunosuppression, the inflammatory response and intercellular communication (59). Tan et al. (60) summarized the antigen-presenting function of exosomes derived from APCs and the immunomodulatory functions of T cellderived exosomes. Exosomes secreted by professional APCs, such as B lymphocytes and DCs, contain MHC I and MHC II complexes and costimulatory molecules. These exosomes have antigenic peptides on the surface, which can play a considerable role in immune regulation $(61,62)$. Recent studies have demonstrated that exosomes are involved in proinflammatory responses and can promote immune responses. Exosomes derived from bacteria- infected macrophages have been shown to have immunomodulatory effects and stimulate macrophages and neutrophils to secrete proinflammatory mediators, including TNF- $\alpha$ and RANTES (upregulating iNOS expression) $(63,64)$. Moreover, exosomes derived from activated $\mathrm{CD} 8^{+} \mathrm{T}$ cells have been demonstrated to express bioactive FasL, Fas and APO2 (apoptin 2) ligands to promote activation-induced cell death that may be required for immune responses (65).

To date, the role of MSC-derived exosomes (MSC-Exos) in immunomodulation is a popular research topic. MSC-Exos have been shown to influence the activities of $\mathrm{B}$ cells and $\mathrm{T}$ cells, stimulate angiogenesis and regulate cell apoptosis and inflammation processes by carrying various immune regulatory factors (cytokines, chemokines and growth factors) (66). For example, studies have shown that MSC-Exos can induce T cell apoptosis through FasL or activate the PD-L1 pathway to inhibit $\mathrm{T}$ cell activation, leading to immune tolerance and inducing the differentiation of Th1 cells into Th2 cells, which then reduces the levels of proinflammatory cytokines, such as IL-1 $\beta$, IL-6, IL-12, and TNF- $\alpha$, and increases the levels of anti-inflammatory

TABLE 1 | Exosomes derived from various cells and their immune roles.

\begin{tabular}{|c|c|c|c|}
\hline Source of exosomes & Exosome cargo & Functions & References \\
\hline \multirow[t]{2}{*}{ B cells } & MHC, CD86, CD54 & Present antigens and activate $\mathrm{CD} 4^{+} \mathrm{T}$ cells & (38) \\
\hline & C3 & Promote $\mathrm{T}$ cells proliferation & (39) \\
\hline LCL & MHC II, FasL & Promotes $\mathrm{CD}^{+}{ }^{+} \mathrm{T}$ cells apoptosis & $(40)$ \\
\hline T cells & DNA & Induce DCs & $(41)$ \\
\hline Activated T cells & FasL, TRAIL & Eliminate the activation of $\mathrm{T}$ cells & (42) \\
\hline \multirow[t]{3}{*}{ Tregs } & Let-7i & Block IGF1R and TGF- $\beta$ R1 pathways & $(43)$ \\
\hline & $\operatorname{miR}-548 a-3 p$ & Interfere with TLR4/NF-кB pathways & (44) \\
\hline & miRNAs & Inhibit Th1 cells proliferation & $(45)$ \\
\hline \multirow[t]{2}{*}{ DCs } & HLA-II & Present antigens & (46) \\
\hline & IL-1, NKG2D & Induce NK cells activation & (46) \\
\hline Mature DCs & MHC, CD86, CD40 & Promotes $T$ cells activation and proliferation & $(47)$ \\
\hline Immature DCs & CD95L & Reduce $T$ cells immune response & $(48)$ \\
\hline \multirow[t]{2}{*}{ Macrophages } & LPS & Induce DCs maturation & (49) \\
\hline & miRNAs & Enhance inflammatory response & $(50,51)$ \\
\hline NK cells & Fas, CD56, NKG2D & Activate immune effector cells and cytotoxicity & (21) \\
\hline \multirow[t]{3}{*}{ MSCs } & PDL1, TGF- $\beta$ & Inhibit T cells immune response & $(52)$ \\
\hline & IL-10, TNF- $\alpha$, IFN- $\gamma$ & Inhibit B cells proliferation & (53) \\
\hline & $\operatorname{miR}-146 a$ & Reduce inflammatory response & (54) \\
\hline MDSCs & & Inhibit T cell proliferation and promote Tregs amplification & (55) \\
\hline
\end{tabular}

LCL, Iymphoblastoid cell line; FasL, human apoptosis-related factor ligand; DCs, dendritic cells; TRAIL, tumor necrosis factor-related apoptosis-inducing ligand; IGF1R, insulin-like growth factor 1 receptor; TGF- $\beta 1 R$, transforming growth factor -beta 1 receptor; TLR4, Toll-like receptor 4; NF- $\kappa B$, nuclear factor $\kappa B$; HLA, human leukocyte antigen; NK cells, natural killer cells; NKG2D, natural killer Group 2 member D; LPS, lipopolysaccharide; PDL1, programmed death ligand 1; IL-10, interleukin-10; TNF- $\alpha$, tumor necrosis factor alpha; IFN- $\gamma$, interferon gamma; MDSCs, myeloid-derived suppressor cells. 
cytokines, such as IL-10 and TGF- $\beta$ (67-69). In addition, MSCExos can also act on macrophages, promoting the polarization of M2 macrophages and the secretion of anti-inflammatory cytokines by macrophages, which helps maintain local immune and metabolic homeostasis (70).

In addition, exosomes contribute significantly to the immunoregulation of Treg cells and Th17 cells. Human umbilical cord MSC-derived exosomes increase the proportion of $\mathrm{CD} 4^{+} \mathrm{CD} 25^{+} \mathrm{Foxp}^{+}$Treg cells and reduce the proportion of $\mathrm{CD}^{+}{ }^{+} \mathrm{IL}_{17 \mathrm{~A}^{+}} \mathrm{T}$ cells (Th17 cells) (71). Moreover, it has been reported that (45) exosome like granules of thymocytes can induce the differentiation of $\mathrm{CD} 4^{+} \mathrm{CD} 25^{-} \mathrm{T}$ cells into $\mathrm{CD} 4^{+} \mathrm{CD} 25^{+} \mathrm{Foxp}^{+}$Treg cells, which contributes to maintaining the immune tolerance of peripheral tissues. In summary, exosomes can affect the differentiation and function of Th cells (T helper cells) and Treg cells, and serve as intercellular mediators that regulate several types of immune responses (Figure 1).

\section{EXOSOMES AND AITDS}

\section{Exosomes and HT}

HT, also known as chronic lymphocytic thyroiditis, is the most common autoimmune thyroid disease, accounting for approximately $22.5 \%$ of thyroid diseases (72). It is characterized by infiltration of thyroid-specific $\mathrm{T}$ lymphocytes and other immune cells, goiter enlargement and fibrosis, destruction of thyroid cells, and eventually hypothyroidism (73). A few HT patients have no goiter, and approximately $50 \%$ of patients have clinical hypothyroidism. The characteristic autoantibodies of HT mainly include TPOAb and $\mathrm{TGAb}$ (74), which have the function of fixing complement, causing cytotoxicity and damaging thyrocytes. In addition, HT patients may also have thyroid stimulating blocking antibodies (TSBAbs) (74), which can promote thyroid atrophy and hypofunction. In HT patients, Th1 cells are the dominant infiltrating lymphocytes, which release cytokines (IFN- $\gamma$, IL-2, and TNF- $\alpha$ ) under the stimulation of thyroid autoantigen, and the latter stimulates the expression of Fas on the surface of the thyroid cells, thereby accelerating the apoptosis of the thyroid cells (75). Exosomes from human B cell-derived lymphoblastoid cell lines highly express MHC II and FasL, which can induce the apoptosis of $\mathrm{CD} 4^{+} \mathrm{T}$ cells through the interaction of FasL and Fas $(40,76,77)$. Therefore, exosomes could be associated with cell apoptosis and participate in several pathological processes related to HT.

Exosomal surface protein markers such as HSP60 and HSP70 can mediate immunomodulatory effects and immune responses (78, 79). HSP60 can bind a variety of receptors present on the surface of immune cells, such as TLRs, CD40, and CD9, leading to antigen cross-presentation, T cell cross-priming, and immune response (78, 80). Cui et al. (81) detected serum exosomes of $40 \mathrm{HT}$ patients and

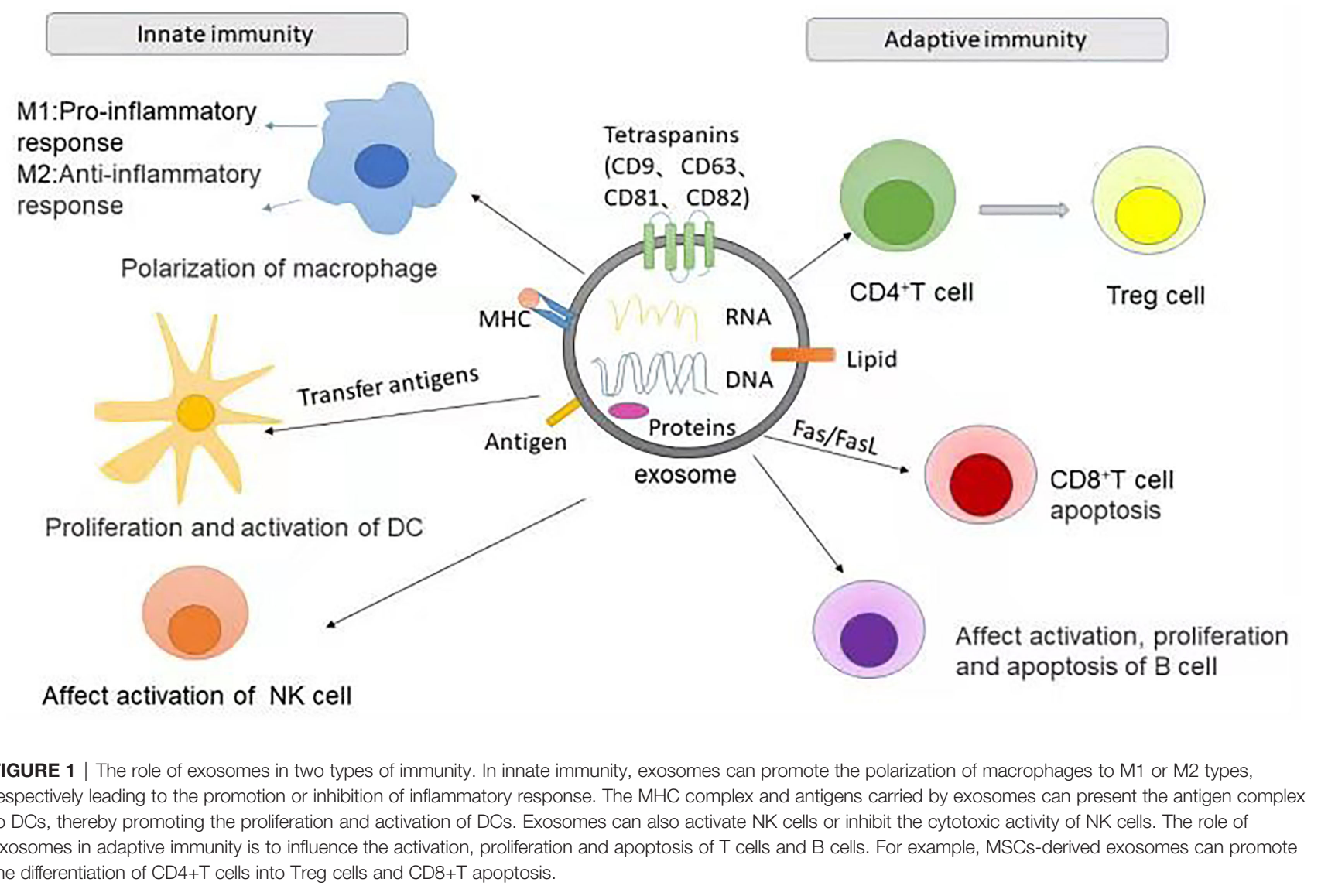


40 healthy controls, and concluded that exosomes from HT patients (HT-Exos) highly expressed the inflammatory factor HSP60, which was positively correlated with serum TPOAb and TgAb. After coculture with healthy human DCs, HT-Exos derived HSP60 was spatially closely arranged and more fully bound to TLR2 on DCs compared with intracellular HSP60. Similarly, another study found that the level of HSP60 in thyroid follicular cell-derived exosomes stimulated by IFN- $\gamma$ was significantly higher than that in normal thyrocyte-derived exosomes. Interestingly, the structure of HSP60 is highly homologous with the structures of TPO and TG, and mediates the disruption of thyrocytes through antigen-antibody reactions in HT (82). Therefore, there is a hypothesis that exosomal HSP60 may serve as an antigen to mediate immunological functions in HT pathogenesis. However, further studies are required to identify the mechanisms of exosomal HSP60-mediated regulation of HT pathogenesis.

Current studies have demonstrated that Th1/Treg, Th2/Treg and Th17/Treg cells in HT patients are out of balance at the transcription factor level, manifesting as the upregulation of Th1, Th2 and Th17 cells, and the downregulation of Treg cells (8385). In this regard, exosomes from HT patients cocultured with peripheral blood mononuclear cells (PBMCs) of healthy humans increased the percentage of $\mathrm{CD} 4^{+}$IFN $-\gamma^{+}$Th1 cells and CD $4^{+} \mathrm{IL}-$ $17 \mathrm{~A}^{+}$Th17A cells in PBMCs, and decreased the percentage of $\mathrm{CD}^{+} \mathrm{CD} 25^{+} \mathrm{Foxp}^{+}$Treg cells in PBMCs (81). Furthermore, this study also found that exosomes from HT patients cocultured with DCs bind to TLR2/3 of DCs, and activate DCs through the TLR2/myeloid differentiation factor 88 (MyD88)/NF- $\mathrm{KB}$ and TLR3/TRIF/NF- $\kappa \mathrm{B}$ pathways, thus leading to an imbalance in the differentiation of $\mathrm{CD} 4{ }^{+} \mathrm{T}$ lymphocytes. In conclusion, these findings support a possible role for exosomes in HT pathogenesis: the levels and composition of circulating exosomes in HT patients could induce T cell imbalance.

As the most powerful professional APCs and $\mathrm{CD}^{+} \mathrm{T}$ lymphocytes, DCs are the main cells involved in the inflammatory response, and they participate in the pathogenesis of HT (86). Research has revealed that APCderived exosomes play a role in antigen presentation and $\mathrm{T}$ lymphocyte activation. For example, mature DC-derived exosomes have been shown to induce the proliferation of $\mathrm{CD} 4^{+} \mathrm{T}$ cells in an antigen-specific manner (87), but the role of exosomes derived from nonprofessional APCs (such as thyroid follicular cells) in these processes is rarely described. Another study by Cui et al. (88) found that thyroid follicular cellderived exosomes stimulated by IFN- $\boldsymbol{\gamma}$ (IFN- $\boldsymbol{\gamma}$-Exos) cocultured with DCs increased the expression of costimulators CD40 and CD80 and the mature marker CD83 on DCs, and increased the gene expression levels of inflammatory cytokines such as IL-6 and TNF. Furthermore, after culturing DCs, IFN- $\boldsymbol{\gamma}$-Exos cocultured with $\mathrm{CD}^{+} \mathrm{T}$ lymphocytes from healthy humans significantly increased the mRNA expression levels of IFN- $\gamma$, IL-17A, IL-22, IL-4, IL-10 and TGF- $\beta 1$ in CD4 ${ }^{+} \mathrm{T}$ cells. All of the evidence suggests that exosomes could activate DCs to further cause an imbalance in cytokine expression and the secretion of $\mathrm{CD}^{+}{ }^{+} \mathrm{T}$ cells, which may lead to the formation of thyroid tissue and a systemic inflammatory environment. These inflammatory microenvironments can further promote the release of "abnormal exosomes" from thyrocytes in vivo, forming a positive feedback loop involved in HT pathogenesis. Additionally, another study observed that the exosome inhibitor GW4869 blocked the activation of DCs by thyroid follicular cells after inhibiting IFN- $\gamma$-Exos, suggesting that exosomes play a key role in antigen presentation and the inflammatory response. GW4869 is a neutral sphingomyelinase inhibitor with cell permeability and selectivity, and it is basically recognized as an exosome inhibitor. It can inhibit the secretion of exosomes in vivo or in vitro, which has been reported in tumors, inflammation and infection (89-92). These studies indicate that intervention in exosome secretion may have a therapeutic effect on inflammation and autoimmune diseases. Together, these results suggest a potential role of thyroid follicular cell-derived exosomes in regulating $\mathrm{T}$ cell differentiation imbalance in the occurrence and development of HT, but the specific mechanism remains to be further clarified.

\section{Exosomes and GD}

GD, namely, diffuse toxic goiter, is an organ-specific autoimmune disease with abnormal thyroid hormone synthesis, accounting for more than $85 \%$ of patients with hyperthyroidism, with a high incidence in women aged 40-60 years old (93). GD mainly manifests as thyrotoxicosis, diffuse goiters and Graves' ophthalmopathy (GO) (94). Abnormal infiltration of $\mathrm{T}$ lymphocytes and production of the autoantibody TRAb are the pathophysiological basis of GD (95). The imbalance of Th1/Th2 cells has become a research focus of GD in recent years. It is generally believed that the inflammatory response mediated by Th1 cells is dominant in the active phase of GD, while Th2 cells mainly play a role in the inactive phase of GD (96). In GD, the recruitment of Th1 lymphocytes leads to increased production of IFN- $\gamma$ and TNF$\alpha$, which stimulates thyroid cells to secrete Th1 chemokines, promoting the autoimmune process. A study (97) investigated the role of IL-21 in the regulation of Th17/Treg cells in 28 newly diagnosed GD patients, 27 remission GD (EGD) patients and 24 healthy subjects and found that IL-21 could stimulate the differentiation of $\mathrm{CD}^{+} \mathrm{T}$ cells into $\mathrm{Th} 17$ cells, reduce the differentiation of Treg cells, and contribute to the activation of the downstream immune response and the pathogenesis of GD.

A number of studies have implicated that IGF-1R and TSHR (thyrotropin receptor) are involved in GD pathogenesis and have a direct interaction in thyroid related eye diseases. Reports from Huang et al. (98) demonstrated that IGF-1R, TPO, TSHR and HSP60 existed in serum exosomes from GD patients and healthy controls. In particular, the levels of IGF-1R and TPO in the serum exosomes of GD patients and active GO patients without hormone shock therapy were significantly higher than those in healthy controls. Further correlation analysis showed that IGF1R was significantly positively correlated with TRAb levels, suggesting that exosomes may present antigens and carry autoantigens (IGF-1R) in GD pathogenesis. However, further study is required to identify why circulating exosomes are specifically recognized as autoantigens in GD. 
Additionally, Rossi and his colleagues observed that the formation and transfer of exosomes from thyroid follicular cells induced by dichlorodiphenyltrichloroethane (DDT) contained thyrotropin-releasing hormone, which could directly stimulate TSHR to binding to autoantibodies, leading to GD development (99). Interestingly, Edo et al. (100) provided evidence that TSHR is detected in exosomes secreted by normal thyroid follicular cell lines and cancerous thyroid cell lines. To examine the function of TSHR exosomes in GD, HEK (human embryonic kidney)/TSHR cells were generated as a model for thyroid follicular epithelial cells in GD where TSHR is upregulated. This study demonstrated that TSHR exosomes isolated from HEK/TSHR cells may exert a decoy effect by binding to and sequestering autoantibodies, thereby ameliorating autoantibody-mediated activation of thyroid function in a GD model. These results seem to contradict each other; therefore, it is necessary to further study the function of TSHR in exosomes in GD.

Circulating exosomes from GD patients were observed to be immunologically active and were capable of inducing the production of inflammatory cytokines from healthy PBMCs. Hiratsuka et al. (101) isolated serum exosomes from patients with refractory GD, and these exosomes stimulated the mRNA expression of inflammatory cytokines such as IL-1, IL-6 and TNF- $\alpha$ in PBMCs, thereby activating the immune response over that found in GD patients in remission or healthy controls. Similarly, Huang et al. (98) uncovered that coculture of serum exosomes from GD patients and healthy human PBMCs promoted the increased expression of $\mathrm{CD} 11 \mathrm{c}^{+} \mathrm{TLR}^{+} \mathrm{DCs}$ and

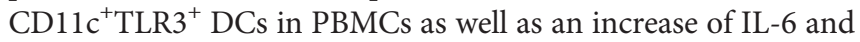
IL- $1 \beta$ in the supernatant. These results suggest that exosomes from GD patients are able to promote the inflammatory response, favoring the pathogenesis and development of GD. To further investigate the specific mechanism of exosomes in promoting the inflammatory response, Cui et al. (102)recently analyzed serum exosomes from 26 healthy controls (HC-Exos), 26 GD patients (GD-Exos) and 7 Graves ophthalmopathy patients (GO-Exos). In this study, after coculture of healthy human PBMCs with GD-Exos or HC-Exos for 24 hours, GDExos could bind to TLR $2 / 3$ and increase the percentage of

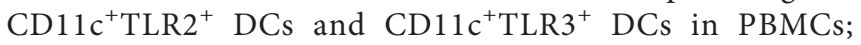
therefore, GD-Exos may mediate their role by acting on TLRs. Furthermore, GD-Exos also significantly increased the protein expression of MyD88, TRIF and p-P65 in PBMCs, as well as the levels of IL- 6 and IL-1 $\beta$ in the medium. It is therefore possible that GD-Exos might participate in GD pathogenesis by binding to TLR2/3 to activate the NF- $\kappa \mathrm{B}$ signaling pathway and induce an inflammatory response. While the mechanisms have not been fully revealed, these observations provide clues that exosomes can promote the inflammatory response in GD.

In addition to synergies between immune factors and environmental stimulation, epigenetics also plays an important role in the pathogenesis of GD, including DNA demethylation, histone modification and noncoding RNAs(ncRNAs) interference $(103,104)$. Intriguingly, the role of noncoding RNAs (including miRNAs, long noncoding RNAs, and
circRNAs) in the etiopathogenesis of GD is increasingly attracting attention $(105,106)$. It has been revealed that nucleic acids contained within exosomes retain their function after exosomal vesicles direct transfer their contents into a recipient cell (107). Research has discovered differences in exosomal noncoding RNAs between healthy populations and GD patients at different stages. Hiratsuka et al. (101) indicated that plasma exosomes from GD patients carry upregulated or downregulated miRNA levels and are associated with disease progression. In this study, compared with intractable GD patients, the levels of miR-23b-5p and miR-92a-3p in serum exosomes of GD patients in remission were significantly increased, while the levels of let-7g-3p and miR-339-5p were significantly decreased. However, the molecular mechanisms of these miRNAs related to GD have not yet been fully elucidated. Other studies have discovered that miRNAs in serum exosomes of intractable GD patients can stimulate mRNA expression of IL$1 \beta$ and TNF- $\alpha$, which may be closely related to the pathogenesis and disease progression of GD. Additionally, Sun et al. (108) showed that plasma exosome circRNAs from GD patients were more than twice as upregulated or downregulated compared with healthy controls, with 15 circRNAs significantly differentially expressed, including the upregulation of 6 circRNAs and the downregulation of 9 circRNAs. This study further verified the significantly increased expression level of has-circRNA-000102 in the plasma exosomes of GD patients and revealed the potential pathways related to GD pathogenesis, namely, herpes simplex virus infection, influenza A signaling and IFN- $\beta$ signaling. These observations imply the potential roles of exosome-derived noncoding RNAs as significant regulators and biomarkers for diagnosing GD. However, more studies are required to investigate exosomal RNAs and their significance in the pathophysiology and diagnosis of GD.

Thyroid ophthalmopathy (TED) is the most common extrathyroid manifestation of AITD. It includes GO, which in severe cases can result in visual field loss due to corneal rupture or compression optic neuropathy (109). Han et al. (110) isolated tear exosomes from GD patients with TED, and found that the tear exosomes of TED patients were 2.3 times higher than those of healthy controls, and highly expressed vitamin $\mathrm{D}$ binding protein (VDBP), C-reactive protein (CRP), chitinase 3-like 1 (CH3L1), matrix metalloproteinase-9 (MMP-9) and vascular adhesion molecule-1 (VCAM-1).

Matrix metalloproteinases play a key role in tissue remodeling in the process of fibrosis and inflammation. They are expressed at very low levels in normal tissues, but can be increased by the influence of inflammatory cytokines, intercellular interactions, hormones, and growth factors (111). Tear derived exosomes can trigger orbital fibroblasts to release the inflammatory cytokines, IL-6, IL-8 and monocyte chemoattractant protein-1 (MCP1) in vitro. These findings suggest that an increased abundance of specific proteins in exosomes can also activate inflammatory responses through orbital fibroblasts (the target cells in TED). In general, increased levels of MMP-9 in tear exosomes could promote orbital tissue remodeling and fibrosis, which contributes to the occurrence and development of TED. 


\section{CONCLUSIONS}

As a newly discovered biological carrier, exosomes contain various proteins, nucleic acids, lipids and other biological information that can regulate the docking and membrane fusion between exosomes and target cells, thus affecting the occurrence and progression of many diseases (112). Recent studies have highlighted the ability of exosomes to regulate immune responses and their great potential as biomarkers for detecting autoimmune diseases. As the most common organ-specific autoimmune disease, AITDs are caused by a complex interaction of genetic and environmental factors. Autoimmune intolerance and $\mathrm{T}$ lymphocyte imbalance are important factors in the pathogenesis of AITDs. It is now known that exosomes can affect the balance of Treg and Th17 cell differentiation and induce inflammatory responses, leading to the breakdown of autoimmune tolerance in AITDs. In addition, exosomes and their cargos from AITD patients are reported to be upregulated and participate in numerous biological processes as well as in AITD pathogenesis. Based on the current findings, the potential application of exosomes as diagnostic biomarkers and

\section{REFERENCES}

1. Tomer Y. Mechanisms of Autoimmune Thyroid Diseases: From Genetics to Epigenetics. Annu Rev Pathol (2014) 9:147-56. doi: 10.1146/annurevpathol-012513-104713

2. Antonelli A, Ferrari SM, Corrado A, Di Domenicantonio A, Fallahi P. Autoimmune Thyroid Disorders. Autoimmun Rev (2015) 14:174-80. doi: 10.1016/j.autrev.2014.10.016

3. Mikoś H, Mikoś M, Obara-Moszyńska M, Niedziela M. The Role of the Immune System and Cytokines Involved in the Pathogenesis of Autoimmune Thyroid Disease (AITD). Endokrynol Pol (2014) 65:150-5. doi: 10.5603/EP.2014.0021

4. Tomer Y, Huber A. The Etiology of Autoimmune Thyroid Disease: A Story of Genes and Environment. J Autoimmun (2009) 32:231-9. doi: 10.1016/ j.jaut.2009.02.007

5. Li Q, Wang B, Mu K, Zhang JA. The Pathogenesis of Thyroid Autoimmune Diseases: New T Lymphocytes - Cytokines Circuits Beyond the Th1-Th2 Paradigm. J Cell Physiol (2019) 234:2204-16. doi: 10.1002/jcp.27180

6. Liang J, Zeng W, Fang F, Yu T, Zhao Y, Fan X, et al. Clinical Analysis of Hashimoto Thyroiditis Coexistent With Papillary Thyroid Cancer in 1392 Patients. Acta Otorhinolaryngol Ital (2017) 37:393-400. doi: 10.14639/0392100X-1709

7. Conigliaro A, Fontana S, Raimondo S, Alessandro R. Exosomes: Nanocarriers of Biological Messages. Adv Exp Med Biol (2017) 998:23-43. doi: 10.1007/978-981-10-4397-0_2

8. Shenoda BB, Ajit SK. Modulation of Immune Responses by Exosomes Derived From Antigen-Presenting Cells. Clin Med Insights Pathol (2016) 9:1-8. doi: 10.4137/CPath.S39925

9. Lee HJ, Li CW, Hammerstad SS, Stefan M, Tomer Y. Immunogenetics of Autoimmune Thyroid Diseases: A Comprehensive Review. J Autoimmun (2015) 64:82-90. doi: 10.1016/j.jaut.2015.07.009

10. Shao S, Yu X, Shen L. Autoimmune Thyroid Diseases and Th17/Treg Lymphocytes. Life Sci (2018) 192:160-5. doi: 10.1016/j.lfs.2017.11.026

11. Rodríguez-Muñoz A, Martínez-Hernández R, Ramos-Leví AM, SerranoSomavilla A, González-Amaro R, Sánchez-Madrid F, et al. Circulating Microvesicles Regulate Treg and Th17 Differentiation in Human Autoimmune Thyroid Disorders. J Clin Endocrinol Metab (2015) 100: E1531-1539. doi: 10.1210/jc.2015-3146

12. Pan BT, Johnstone RM. Fate of the Transferrin Receptor During Maturation of Sheep Reticulocytes In Vitro: Selective Externalization of the Receptor. Cell (1983) 33:967-78. doi: 10.1016/0092-8674(83)90040-5 therapeutics for AITDs is a future opportunity. However, the basic and applied research on exosomes is still in its early stage, and the potential mechanism in AITDs has not been fully clarified. Consequently, continued research is required to elucidate the exact mechanism of exosomes in AITDs, thus contributing to a deeper understanding of disease diagnosis and prognosis.

\section{AUTHOR CONTRIBUTIONS}

JZ drafted the manuscript. HP and YL designed the study and revised the manuscript. All authors contributed to the article and approved the submitted version.

\section{FUNDING}

This work was supported by National Natural Science Foundation of China (Grant No. 81800698), Zhenjiang Science and Technology Planning Project (Grant No. SH2021026, SH2021059).

13. Lobb RJ, Becker M, Wen SW, Wong CS, Wiegmans AP, Leimgruber A, et al Optimized Exosome Isolation Protocol for Cell Culture Supernatant and Human Plasma. J Extracell Vesicles (2015) 4:27031. doi: 10.3402/jev.v4. 27031

14. Gurunathan S, Kang MH, Jeyaraj M, Qasim M, Kim JH. Review of the Isolation, Characterization, Biological Function, and Multifarious Therapeutic Approaches of Exosomes. Cells (2019) 8(4):307. doi: 10.3390/ cells 8040307

15. Li P, Kaslan M, Lee SH, Yao J, Gao Z. Progress in Exosome Isolation Techniques. Theranostics (2017) 7:789-804. doi: 10.7150/thno.18133

16. Cui S, Cheng Z, Qin W, Jiang L. Exosomes as a Liquid Biopsy for Lung Cancer. Lung Cancer (2018) 116:46-54. doi: 10.1016/j.lungcan.2017.12.012

17. Rashed HR, Bayraktar E, Helal KG, Abd-Ellah MF, Amero P, Chavez-Reyes A, et al. Exosomes: From Garbage Bins to Promising Therapeutic Targets. Int J Mol Sci (2017) 18(3):538. doi: 10.3390/ijms18030538

18. Mathivanan S, Ji H, Simpson RJ. Exosomes: Extracellular Organelles Important in Intercellular Communication. J Proteomics (2010) 73:190720. doi: 10.1016/j.jprot.2010.06.006

19. van Niel G, Porto-Carreiro I, Simoes S, Raposo G. Exosomes: A Common Pathway for a Specialized Function. J Biochem (2006) 140:13-21. doi: 10.1093/ $\mathrm{jb} / \mathrm{mvj} 128$

20. Mashouri L, Yousefi H, Aref AR, Ahadi AM, Molaei F, Alahari SK. Exosomes: Composition, Biogenesis, and Mechanisms in Cancer Metastasis and Drug Resistance. Mol Cancer (2019) 18:75. doi: 10.1186/s12943-019-0991-5

21. Jeppesen DK, Fenix AM, Franklin JL, Higginbotham JN, Zhang Q, Zimmerman LJ, et al. Reassessment of Exosome Composition. Cell (2019) 177:428-45.e418. doi: 10.1016/j.cell.2019.02.029

22. Sahoo S, Losordo DW. Exosomes and Cardiac Repair After Myocardial Infarction. Circ Res (2014) 114:333-44. doi: 10.1161/CIRCRESAHA.114.300639

23. Zhao T, Sun F, Liu J, Ding T, She J, Mao F, et al. Emerging Role of Mesenchymal Stem Cell-Derived Exosomes in Regenerative Medicine. Curr Stem Cell Res Ther (2019) 14:482-94. doi: 10.2174/1574888X14666190228103230

24. Kharaziha P, Ceder S, Li Q, Panaretakis T. Tumor Cell-Derived Exosomes: A Message in a Bottle. Biochim Biophys Acta (2012) 1826:103-11. doi: 10.1016/ j.bbcan.2012.03.006

25. Keller S, Sanderson MP, Stoeck A, Altevogt P. Exosomes: From Biogenesis and Secretion to Biological Function. Immunol Lett (2006) 107:102-8. doi: 10.1016/j.imlet.2006.09.005

26. Gallo A, Tandon M, Alevizos I, Illei GG. The Majority of microRNAs Detectable in Serum and Saliva is Concentrated in Exosomes. PLoS One (2012) 7:e30679. doi: 10.1371/journal.pone.0030679 
27. Yang C, Robbins PD. The Roles of Tumor-Derived Exosomes in Cancer Pathogenesis. Clin Dev Immunol (2011) 2011:842849. doi: 10.1155/2011/842849

28. Shan SK, Lin X, Li F, Xu F, Zhong JY, et al. Exosomes and Bone Disease. Curr Pharm Des (2019) 25:4536-49. doi: 10.2174/1381612825666191127114054

29. Chen JQ HL. Advances in the Molecular Mechanism of Mesenchymal Stem Cells and Their Exosomes Promoting Angiogenesis. Chin J Exp Hematol (2018) 26:1858-62. doi: 10.7534/j.issn.1009-2137.2018.06.047

30. Gong M, Yu B, Wang J, Wang Y, Liu M, Paul C, et al. Mesenchymal Stem Cells Release Exosomes That Transfer miRNAs to Endothelial Cells and Promote Angiogenesis. Oncotarget (2017) 8:45200-12. doi: 10.18632/ oncotarget.16778

31. Sadri Nahand J, Moghoofei M, Salmaninejad A, Bahmanpour Z, Karimzadeh M, Nasiri M, et al. Pathogenic Role of Exosomes and microRNAs in HPV-Mediated Inflammation and Cervical Cancer: A Review. Int J Cancer (2020) 146:305-20. doi: 10.1002/ijc.32688

32. Zhang S, Chuah SJ, Lai RC, Hui JHP, Lim SK, Toh WS. MSC Exosomes Mediate Cartilage Repair by Enhancing Proliferation, Attenuating Apoptosis and Modulating Immune Reactivity. Biomaterials (2018) 156:16-27. doi: 10.1016/j.biomaterials.2017.11.028

33. Yáñez-Mó M, Siljander PR, Andreu Z, Zavec AB, Borràs FE, Buzas EI, et al. Biological Properties of Extracellular Vesicles and Their Physiological Functions. J Extracell Vesicles (2015) 4:27066. doi: 10.3402/jev.v4.27066

34. Théry C, Zitvogel L, Amigorena S. Exosomes: Composition, Biogenesis and Function. Nat Rev Immunol (2002) 2:569-79. doi: 10.1038/nri855

35. Okoye IS, Coomes SM, Pelly VS, Czieso S, Papayannopoulos V, Tolmachova T, et al. MicroRNA-Containing T-Regulatory-Cell-Derived Exosomes Suppress Pathogenic T Helper 1 Cells. Immunity (2014) 41:89-103. doi: 10.1016/j.immuni.2014.05.019

36. Peng MY, He H, Wang XW, Cai CC, Liu X, Xu ZJ, et al. Advances in Biological Functions and Regulatory Mechanisms of Exosomes. Chin J Anim Sci (2021) 57:11-6. doi: 10.19556/j.0258-7033.20200224-01

37. Hwang I. Cell-Cell Communication via Extracellular Membrane Vesicles and its Role in the Immune Response. Mol Cells (2013) 36:105-11. doi: 10.1007/s10059-013-0154-2

38. Ren Y, Yang J, Xie R, Gao L, Yang Y, Fan H, et al. Exosomal-Like Vesicles With Immune-Modulatory Features are Present in Human Plasma and can Induce CD4+ T-Cell Apoptosis in vitro. Transfusion (2011) 51:1002-11. doi: 10.1111/j.1537-2995.2010.02909.x

39. Papp K, Végh P, Prechl J, Kerekes K, Kovács J, Csikós G, et al. B Lymphocytes and Macrophages Release Cell Membrane Deposited C3Fragments on Exosomes With T Cell Response-Enhancing Capacity. Mol Immunol (2008) 45:2343-51. doi: 10.1016/j.molimm.2007.11.021

40. Lema DA, Burlingham WJ. Role of Exosomes in Tumour and Transplant Immune Regulation. Scand J Immunol (2019) 90:e12807. doi: 10.1111/ sji.12807

41. Torralba D, Baixauli F, Villarroya-Beltri C, Fernández-Delgado I, LatorrePellicer A, Acín-Pérez R, et al. Priming of Dendritic Cells by DNAContaining Extracellular Vesicles From Activated T Cells Through Antigen-Driven Contacts. Nat Commun (2018) 9:2658. doi: 10.1038/ s41467-018-05077-9

42. Xu R, Rai A, Chen M, Suwakulsiri W, Greening DW, Simpson RJ. Extracellular Vesicles in Cancer - Implications for Future Improvements in Cancer Care. Nat Rev Clin Oncol (2018) 15:617-38. doi: 10.1038/s41571018-0036-9

43. Kimura K, Hohjoh H, Fukuoka M, Sato W, Oki S, Tomi C, et al. Circulating Exosomes Suppress the Induction of Regulatory T Cells via let-7i in multiple sclerosis. Nat Commun (2018) 9:17. doi: 10.1038/s41467-017-02406-2

44. Wang Y, Zheng F, Gao G, Yan S, Zhang L, Wang L, et al. MiR-548a-3p Regulates Inflammatory Response via TLR4/NF- $\mathrm{bb}$ Signaling Pathway in Rheumatoid Arthritis. J Cell Biochem (2018). doi: 10.1002/jcb.26659

45. Chatila TA, Williams CB. Regulatory T Cells: Exosomes Deliver Tolerance. Immunity (2014) 41:3-5. doi: 10.1016/j.immuni.2014.07.001

46. Buschow SI, Nolte-'t Hoen EN, van Niel G, Pols MS, ten Broeke T, Lauwen M, et al. MHC II in Dendritic Cells is Targeted to Lysosomes or T CellInduced Exosomes via Distinct Multivesicular Body Pathways. Traffic (2009) 10:1528-42. doi: 10.1111/j.1600-0854.2009.00963.x

47. Gao W, Liu H, Yuan J, Wu C, Huang D, Ma Y, et al. Exosomes Derived From Mature Dendritic Cells Increase Endothelial Inflammation and
Atherosclerosis via Membrane TNF- $\alpha$ Mediated NF- $\kappa b$ Pathway. J Cell Mol Med (2016) 20:2318-27. doi: 10.1111/jcmm.12923

48. Pêche H, Renaudin K, Beriou G, Merieau E, Amigorena S, Cuturi MC. Induction of Tolerance by Exosomes and Short-Term Immunosuppression in a Fully MHC-Mismatched Rat Cardiac Allograft Model. Am J Transplant (2006) 6:1541-50. doi: 10.1111/j.1600-6143.2006.01344.x

49. O'Neill HC, Quah BJ. Exosomes Secreted by Bacterially Infected Macrophages are Proinflammatory. Sci Signal (2008) 1:pe8. doi: 10.1126/ stke.16pe8

50. Goto Y, Ogawa Y, Tsumoto H, Miura Y, Nakamura TJ, Ogawa K, et al. Contribution of the Exosome-Associated Form of Secreted Endoplasmic Reticulum Aminopeptidase 1 to Exosome-Mediated Macrophage Activation. Biochim Biophys Acta Mol Cell Res (2018) 1865:874-88. doi: 10.1016/j.bbamcr.2018.03.009

51. McDonald MK, Tian Y, Qureshi RA, Gormley M, Ertel A, Gao R, et al. Functional Significance of Macrophage-Derived Exosomes in Inflammation and Pain. Pain (2014) 155:1527-39. doi: 10.1016/j.pain.2014.04.029

52. LIU M-Y, F L, Zhang W-H, Zhang L-B. Progress in Mechanism of Interaction Between Immune Cells and Exosomes. Chin J Immunol (2019) 35:2806-12. doi: 10.3969/j.issn.1000-484X.2019.22.024

53. Wu NX, Yan CX, Fu Y. Effect of Exosomes on Immunomodulatory Regulation and Research Progress. Chin J Cell Mol Immunol (2018) 34:273-7. doi: 10.13423/j.cnki.cjcmi.008572

54. Sun G, Li G, Li D, Huang W, Zhang R, Zhang H, et al. hucMSC Derived Exosomes Promote Functional Recovery in Spinal Cord Injury Mice via Attenuating Inflammation. Mater Sci Eng C Mater Biol Appl (2018) 89:194204. doi: 10.1016/j.msec.2018.04.006

55. Zhou M, Chen J, Zhou L, Chen W, Ding G, Cao L. Pancreatic Cancer Derived Exosomes Regulate the Expression of TLR4 in Dendritic Cells via miR-203. Cell Immunol (2014) 292:65-9. doi: 10.1016/j.cellimm.2014.09.004

56. Li Q, Huang Q, Huyan T, Wang Y, Huang Q, Shi J. Bifacial Effects of Engineering Tumour Cell-Derived Exosomes on Human Natural Killer Cells. Exp Cell Res (2018) 363:141-50. doi: 10.1016/j.yexcr.2017.12.005

57. Borrelli C, Ricci B, Vulpis E, Fionda C, Ricciardi MR, Petrucci MT, et al. Drug-Induced Senescent Multiple Myeloma Cells Elicit NK Cell Proliferation by Direct or Exosome-Mediated IL15 Trans-Presentation. Cancer Immunol Res (2018) 6:860-9. doi: 10.1158/2326-6066.CIR-17-0604

58. Bobrie A, Colombo M, Raposo G, Théry C. Exosome Secretion: Molecular Mechanisms and Roles in Immune Responses. Traffic (2011) 12:1659-68. doi: 10.1111/j.1600-0854.2011.01225.x

59. Greening DW, Gopal SK, Xu R, Simpson RJ, Chen W. Exosomes and Their Roles in Immune Regulation and Cancer. Semin Cell Dev Biol (2015) 40:7281. doi: 10.1016/j.semcdb.2015.02.009

60. Tan L, Wu H, Liu Y, Zhao M, Li D, Lu Q. Recent Advances of Exosomes in Immune Modulation and Autoimmune Diseases. Autoimmunity (2016) 49:357-65. doi: 10.1080/08916934.2016.1191477

61. Lindenbergh MFS, Stoorvogel W. Antigen Presentation by Extracellular Vesicles From Professional Antigen-Presenting Cells. Annu Rev Immunol (2018) 36:435-59. doi: 10.1146/annurev-immunol-041015-055700

62. Beach A, Zhang HG, Ratajczak MZ, Kakar SS. Exosomes: An Overview of Biogenesis, Composition and Role in Ovarian Cancer. J Ovarian Res (2014) 7:14. doi: 10.1186/1757-2215-7-14

63. Bhatnagar S, Schorey JS. Exosomes Released From Infected Macrophages Contain Mycobacterium Avium Glycopeptidolipids and are Proinflammatory. J Biol Chem (2007) 282:25779-89. doi: 10.1074/jbc.M702277200

64. Bhatnagar S, Shinagawa K, Castellino FJ, Schorey JS. Exosomes Released From Macrophages Infected With Intracellular Pathogens Stimulate a Proinflammatory Response In Vitro and In Vivo. Blood (2007) 110:323444. doi: 10.1182/blood-2007-03-079152

65. Monleón I, Martínez-Lorenzo MJ, Monteagudo L, Lasierra P, Taulés M, Iturralde $\mathrm{M}$, et al. Differential Secretion of Fas Ligand- or APO2 Ligand/ TNF-Related Apoptosis-Inducing Ligand-Carrying Microvesicles During Activation-Induced Death of Human T Cells. J Immunol (2001) 167:673644. doi: 10.4049/jimmunol.167.12.6736

66. Tsuchiya A, Kojima Y, Ikarashi S, Seino S, Watanabe Y, Kawata Y, et al. Clinical Trials Using Mesenchymal Stem Cells in Liver Diseases and Inflammatory Bowel Diseases. Inflammation Regener (2017) 37:16. doi: 10.1186/s41232017-0045-6 
67. Harrell CR, Jovicic N, Djonov V, Arsenijevic N, Volarevic V. Mesenchymal Stem Cell-Derived Exosomes and Other Extracellular Vesicles as New Remedies in the Therapy of Inflammatory Diseases. Cells (2019) 8 (12):1605. doi: 10.3390/cells8121605

68. Zhang B, Yin Y, Lai RC, Tan SS, Choo AB, Lim SK. Mesenchymal Stem Cells Secrete Immunologically Active Exosomes. Stem Cells Dev (2014) 23:123344. doi: $10.1089 /$ scd.2013.0479

69. Chen W, Huang Y, Han J, Yu L, Li Y, Lu Z, et al. Immunomodulatory Effects of Mesenchymal Stromal Cells-Derived Exosome. Immunol Res (2016) 64:831-40. doi: 10.1007/s12026-016-8798-6

70. Lumeng CN, Bodzin JL, Saltiel AR. Obesity Induces a Phenotypic Switch in Adipose Tissue Macrophage Polarization. J Clin Invest (2007) 117:175-84. doi: $10.1172 /$ JCI29881

71. Liu M, Wang J, Liu M, Hu X, Xu J. [Study of Immunomodulatory Function of Exosomes Derived From Human Umbilical Cord Mesenchymal Stem Cells]. Zhonghua Yi Xue Za Zhi (2015) 95:2630-3. doi: 10.3760/cma.j.issn. 0376-2491.2015.32.014

72. Dayan CM, Daniels GH. Chronic Autoimmune Thyroiditis. N Engl J Med (1996) 335:99-107. doi: 10.1056/NEJM199607113350206

73. Paknys G, Kondrotas AJ, Kevelaitis E. [Risk Factors and Pathogenesis of Hashimoto's Thyroiditis]. Medicina (Kaunas) (2009) 45:574-83. doi: 10.3390/ medicina45070076

74. Wufuer Y, Wang HG, Halimulati, Li XX. The Differential Diagnostic Value of TSH and TPOAb Levels in Hashimoto's Thyroiditis and Papillary Thyroid Carcinoma. Chin J Difficult Complicated cases (2018) 17:387-90. doi: 10.3969/j.issn.1671-6450.2018.04.015

75. Mezosi E, Wang SH, Utsugi S, Bajnok L, Bretz JD, Gauger PG, et al. Induction and Regulation of Fas-Mediated Apoptosis in Human Thyroid Epithelial Cells. Mol Endocrinol (2005) 19:804-11. doi: 10.1210/me.20040286

76. Klinker MW, Lizzio V, Reed TJ, Fox DA, Lundy SK. Human B Cell-Derived Lymphoblastoid Cell Lines Constitutively Produce Fas Ligand and Secrete MHCII(+)FasL(+) Killer Exosomes. Front Immunol (2014) 5:144. doi: 10.3389/fimmu.2014.00144

77. Li C, Donninger H, Eaton J, Yaddanapudi K. Regulatory Role of Immune Cell-Derived Extracellular Vesicles in Cancer: The Message Is in the Envelope. Front Immunol (2020) 11:1525. doi: 10.3389/fimmu.2020.01525

78. Taha EA, Ono K, Eguchi T. Roles of Extracellular HSPs as Biomarkers in Immune Surveillance and Immune Evasion. Int J Mol Sci (2019) 20 (18):4588. doi: 10.3390/ijms20184588

79. De Maio A, Vazquez D. Extracellular Heat Shock Proteins: A New Location, a New Function. Shock (2013) 40:239-46. doi: 10.1097/SHK. 0b013e3182a185ab

80. Caruso Bavisotto C, Cappello F, Macario AJL, Conway de Macario E, Logozzi M, Fais S, et al. Exosomal HSP60: A Potentially Useful Biomarker for Diagnosis, Assessing Prognosis, and Monitoring Response to Treatment. Expert Rev Mol Diagn (2017) 17:815-22. doi: 10.1080/14737159.2017. 1356230

81. Cui X, Liu Y, Wang S, Zhao N, Qin J, Li Y, et al. Circulating Exosomes Activate Dendritic Cells and Induce Unbalanced CD4+ T Cell Differentiation in Hashimoto Thyroiditis. J Clin Endocrinol Metab (2019) 104:4607-18. doi: 10.1210/jc.2019-00273

82. Marino Gammazza A, Rizzo M, Citarrella R, Rappa F, Campanella C, Bucchieri F, et al. Elevated Blood Hsp60, its Structural Similarities and Cross-Reactivity With Thyroid Molecules, and its Presence on the Plasma Membrane of Oncocytes Point to the Chaperonin as an Immunopathogenic Factor in Hashimoto's Thyroiditis. Cell Stress Chaperones (2014) 19:343-53. doi: 10.1007/s12192-013-0460-9

83. Xue H, Yu X, Ma L, Song S, Li Y, Zhang H, et al. The Possible Role of $\mathrm{CD} 4^{+} \mathrm{CD} 25$ (high)Foxp $3^{+} / \mathrm{CD} 4^{+} \mathrm{IL}-17 \mathrm{~A}^{+}$Cell Imbalance in the Autoimmunity of Patients With Hashimoto Thyroiditis. Endocrine (2015) 50:665-73. doi: 10.1007/s12020-015-0569-y

84. Safdari V, Alijani E, Nemati M, Jafarzadeh A. Imbalances in T Cell-Related Transcription Factors Among Patients With Hashimoto's Thyroiditis. Sultan Qaboos Univ Med J (2017) 17:e174-80. doi: 10.18295/squmj. 2016.17.02.007

85. Figueroa-Vega N, Alfonso-Pérez M, Benedicto I, Sánchez-Madrid F, González-Amaro R, Marazuela M. Increased Circulating Proinflammatory
Cytokines and Thl7 Lymphocytes in Hashimoto's Thyroiditis. J Clin Endocrinol Metab (2010) 95:953-62. doi: 10.1210/jc.2009-1719

86. Ramos-Leví AM, Marazuela M. Pathogenesis of Thyroid Autoimmune Disease: The Role of Cellular Mechanisms. Endocrinol Nutr (2016) 63:421-9. doi: 10.1016/j.endonu.2016.04.003

87. Segura E, Nicco C, Lombard B, Véron P, Raposo G, Batteux F, et al. ICAM-1 on Exosomes From Mature Dendritic Cells is Critical for Efficient Naive TCell Priming. Blood (2005) 106:216-23. doi: 10.1182/blood-2005-01-0220

88. Cui X, Wang S, Zhao N, Wang S, Wang Z, Huang M, et al. Thyrocyte-Derived Exosome-Targeted Dendritic Cells Stimulate Strong CD4(+) T Lymphocyte Responses. Mol Cell Endocrinol (2020) 506:110756. doi: 10.1016/j.mce. 2020.110756

89. Trajkovic K, Hsu C, Chiantia S, Rajendran L, Wenzel D, Wieland F, et al. Ceramide Triggers Budding of Exosome Vesicles Into Multivesicular Endosomes. Science (2008) 319:1244-7. doi: 10.1126/science.1153124

90. Essandoh K, Yang L, Wang X, Huang W, Qin D, Hao J, et al. Blockade of Exosome Generation With GW4869 Dampens the Sepsis-Induced Inflammation and Cardiac Dysfunction. Biochim Biophys Acta (2015) 1852:2362-71. doi: 10.1016/j.bbadis.2015.08.010

91. Lallemand T, Rouahi M, Swiader A, Grazide MH, Geoffre N, Alayrac P, et al. Nsmase2 (Type 2-Neutral Sphingomyelinase) Deficiency or Inhibition by GW4869 Reduces Inflammation and Atherosclerosis in Apoe(-/-) Mice. Arterioscler Thromb Vasc Biol (2018) 38:1479-92. doi: 10.1161/ATVBAHA. 118.311208

92. Gon Y, Maruoka S, Inoue T, Kuroda K, Yamagishi K, Kozu Y, et al. Selective Release of miRNAs via Extracellular Vesicles is Associated With House-Dust Mite Allergen-Induced Airway Inflammation. Clin Exp Allergy (2017) 47:1586-98. doi: 10.1111/cea.13016

93. Smith TJ, Hegedüs L. Graves' Disease. N Engl J Med (2016) 375:1552-65. doi: 10.1056/NEJMra1510030

94. McLachlan SM, Nagayama Y, Rapoport B. Insight Into Graves' Hyperthyroidism From Animal Models. Endocr Rev (2005) 26:800-32. doi: 10.1210/er.2004-0023

95. Schott M, Scherbaum WA, Morgenthaler NG. Thyrotropin Receptor Autoantibodies in Graves' Disease. Trends Endocrinol Metab (2005) 16:243-8. doi: 10.1016/j.tem.2005.05.009

96. Fallahi P, Ferrari SM, Ragusa F, Ruffilli I, Elia G, Paparo SR, et al. Th1 Chemokines in Autoimmune Endocrine Disorders. J Clin Endocrinol Metab (2020) 105(4):dgz289. doi: 10.1210/clinem/dgz289

97. Tan Y, Chen W, Liu C, Zheng X, Guo A, Long J. Effect of IL-21 on the Balance of Th17 Cells/Treg Cells in the Pathogenesis of Graves' Disease. Endocr Res (2019) 44:138-47. doi: 10.1080/07435800.2019.1600535

98. Huang MS, Li YZ. Serum Exosomes in Patients With Graves' Disease Mediate Immune and Inflammatory Responses Through Toll Receptors. China Med Univ (2019), 1-59. doi: 10.27652/d.cnki.gzyku.2019.000800

99. Rossi M, Taddei AR, Fasciani I, Maggio R, Giorgi F. The Cell Biology of the Thyroid-Disrupting Mechanism of Dichlorodiphenyltrichloroethane (DDT). J Endocrinol Invest (2018) 41:67-73. doi: 10.1007/s40618-0170716-9

100. Edo N, Kawakami K, Fujita Y, Morita K, Uno K, Tsukamoto K, et al. Exosomes Expressing Thyrotropin Receptor Attenuate AutoantibodyMediated Stimulation of Cyclic Adenosine Monophosphate Production. Thyroid (2019) 29:1012-7. doi: 10.1089/thy.2018.0772

101. Hiratsuka I, Yamada H, Munetsuna E, Hashimoto S, Itoh M. Circulating MicroRNAs in Graves' Disease in Relation to Clinical Activity. Thyroid (2016) 26:1431-40. doi: 10.1089/thy.2016.0062

102. Cui X, Huang M, Wang S, Zhao N, Huang T, Wang Z, et al. Circulating Exosomes From Patients With Graves' Disease Induce an Inflammatory Immune Response. Endocrinology (2021) 162(3):bqaa236. doi: 10.1210/ endocr/bqaa236

103. Cañas CA, Cañas F, Bonilla-Abadía F, Ospina FE, Tobón GJ. Epigenetics Changes Associated to Environmental Triggers in Autoimmunity. Autoimmunity (2016) 49:1-11. doi: 10.3109/08916934.2015.1086996

104. Wang B, Shao X, Song R, Xu D, Zhang JA. The Emerging Role of Epigenetics in Autoimmune Thyroid Diseases. Front Immunol (2017) 8:396. doi: 10.3389/fimmu.2017.00396

105. Martínez-Hernández R, Serrano-Somavilla A, Ramos-Leví A, Sampedro-Nuñez M, Lens-Pardo A, Muñoz De Nova JL, et al. Integrated miRNA and mRNA Expression 
Profiling Identifies Novel Targets and Pathological Mechanisms in Autoimmune Thyroid Diseases. EBioMedicine (2019) 50:329-42. doi: 10.1016/j.ebiom.2019.10.061

106. Yin L, Zeng C, Yao J, Shen J. Emerging Roles for Noncoding RNAs in Autoimmune Thyroid Disease. Endocrinology (2020) 161(8):bqaa053. doi: 10.1210/endocr/bqaa053

107. Zhu T, Wang Y, Jin H, Li L. The Role of Exosome in Autoimmune Connective Tissue Disease. Ann Med (2019) 51:101-8. doi: 10.1080/07853890.2019.1592215

108. Sun Y, Wang W, Tang Y, Wang D, Li L, Na M, et al. Microarray Profiling and Functional Analysis of Differentially Expressed Plasma Exosomal Circular RNAs in Graves' Disease. Biol Res (2020) 53:32. doi: 10.1186/s40659-020-00299-y

109. Hodgson NM, Rajaii F. Current Understanding of the Progression and Management of Thyroid Associated Orbitopathy: A Systematic Review. Ophthalmol Ther (2020) 9:21-33. doi: 10.1007/s40123-019-00226-9

110. Han JS, Kim Se, Jin JQ, Park NR, Lee JY, Kim HL, et al. Tear-Derived Exosome Proteins Are Increased in Patients With Thyroid Eye Disease. Int J Mol Sci (2021) 22(3):1115. doi: 10.3390/ijms22031115

111. Hemmann S, Graf J, Roderfeld M, Roeb E. Expression of MMPs and TIMPs in Liver Fibrosis - a Systematic Review With Special Emphasis on AntiFibrotic Strategies. J Hepatol (2007) 46:955-75. doi: 10.1016/j.jhep. 2007.02.003
112. Farooqi AA, Desai NN, Qureshi MZ, Librelotto DRN, Gasparri ML, Bishayee A, et al. Exosome Biogenesis, Bioactivities and Functions as New Delivery Systems of Natural Compounds. Biotechnol Adv (2018) 36:328-34. doi: 10.1016/j.biotechadv.2017.12.010

Conflict of Interest: The authors declare that the research was conducted in the absence of any commercial or financial relationships that could be construed as a potential conflict of interest.

Publisher's Note: All claims expressed in this article are solely those of the authors and do not necessarily represent those of their affiliated organizations, or those of the publisher, the editors and the reviewers. Any product that may be evaluated in this article, or claim that may be made by its manufacturer, is not guaranteed or endorsed by the publisher.

Copyright (C) 2021 Zou, Peng and Liu. This is an open-access article distributed under the terms of the Creative Commons Attribution License (CC BY). The use, distribution or reproduction in other forums is permitted, provided the original author(s) and the copyright owner(s) are credited and that the original publication in this journal is cited, in accordance with accepted academic practice. No use, distribution or reproduction is permitted which does not comply with these terms. 